\title{
Atom-Triatom Rigid Rotor Inelastic Scattering with the MultiConfiguration Time Dependent Hartree approach
}

\author{
Steve Ndengué, Richard Dawes \\ Department of Chemistry, Missouri University of Science and Technology, Rolla, MO, 65401, USA. \\ Fabien Gatti \\ Institut des Sciences Moleculaires d'Orsay, CNRS, Université Paris-Sud, Université Paris Saclay, F-91405 Orsay, France. \\ Hans-Dieter Meyer \\ Theoretische Chemie, Physikalisch-Chemisches Institut, Universität Heidelberg, Im Neuenheimer Feld 229, D-69120 Heidelberg, Germany.
}

\begin{abstract}
The inelastic scattering between a rigid rotor triatomic molecule and an atom is described within the frame of the MultiConfiguration Time dependent Hartree (MCTDH) method. Sample calculations are done on the $\mathrm{H}_{2} \mathrm{O}-\mathrm{Ar}$ system for which a flexible 6D PES (used here in the rigid rotor approximation) has been recently computed in our group and will be presented separately. The results are compared with corresponding time independent calculations using the Arthurs and Dalgarno approach and confirm as expected the equivalence of the two methods.
\end{abstract}

Keywords: Quantum dynamics, Inelastic scattering, Time-Dependent, Close-coupling

\section{Introduction}

Collisional (vibrational and rotational) energy transfer has a central role in chemical and physical studies of atomic and molecular species. From a fundamental stand5 point, scattering studies are often used concurrently with spectroscopy to identify signatures of atoms and molecules ${ }_{35}$ in various environments. Scattering dynamics govern transport processes and both reactive and non-reactive scattering are important in kinetics. The importance of vibra10 tional energy transfer [1] is well known for processes such as combustion, laser operation, photochemical reactions and even surface etching. Rotational energy transfer is a key process in many astrophysical environments, competing with radiative processes in the interstellar medium, in15 terstellar clouds, and cool planetary atmospheres. Energy transfer mechanisms control the processes of collisional ${ }_{45}$ stabilization/activation, maintenance of thermal equilibrium and even pyrolysis, all of which are important to chemical reactivity 2 .

The most rigorous approach to obtain information on energy transfer mechanisms is via solution of the quantum ${ }_{50}$ mechanical Schrödinger equation for the atom(s) and/or molecule(s) involved in the process. Within the Born Oppenheimer approximation, one usually solves the electronic 25 Schrödinger equation to provide the potential energy surface (PES) on which the dynamics of the nuclei are studied. While for small systems, the electronic structure part is generally solved quantum mechanically, the dynamics of the nuclei are often treated more approximately (classically or semi-classically). A major issue for quantum dynamics calculations is what is known as 'the curse of dimensionality', or very poor scaling. Quantum dynamics calculations of inelastic collisions are typically very computationally costly, and as a result most of the calculations that have been reported for 'heavy-heavy' collisions (beyond the lightest few-electron systems) were only done fully quantum mechanically either for diatomic systems or for polyatomics at very low energy. Most other calculations made use of mixed quantum/classical, semi-classical or even classical approaches to study collisions at higher energies. In this respect, there has recently been a revival of quantum/classical approaches 3] (where the collisional coordinate is treated classically while the internal modes of the collision partners are described quantum mechanically) to study inelastic collisions, aiming to provide reliable estimates of cross-sections and rates for a large range of systems that have not been feasible because of computational limitations. Babikov's results 3] are very impressive even for some light systems that one might assume would require a fully quantum treatment. However, there are still some limitations to mixed quantum/classical methods particularly when resonances are important. The work presented here explores a fully quantum mechanical approach to providing inelastic cross-sections and rates using alternate methods with improved efficiency to describe systems that have been difficult to study because of the computational cost [4. 
The Multiconfiguration Time Dependent Hartree method has proven in several studies to be able to simulate with 90 good accuracy the spectroscopy (bound states or resonances $[5$ and dynamics (reactive [6, 7, 8] and non-reactive collisions 9 , 10, 11, 12]) of small to medium size systems. The purpose of this work is to demonstrate the capability of MCTDH to accurately treat the inelastic scattering of an asymmetric 95 rigid triatomic molecule with a heavy atom: the cases of spherical or symmetric tops could be treated more simply.

The paper is organized as follows: Section 2 is a brief description of the MCTDH method; Section 3 details the steps to obtain atom-triatom inelastic cross-sections with the MCTDH approach and how it is applied to the scattering of $\mathrm{H}_{2} \mathrm{O}$ with Ar. We end the paper with concluding ${ }_{100}$ remarks addressing its potential applications and further extensions.

\section{The MCTDH method}

The Multiconfiguration Time Dependent Hartree 13, 14. 15. (MCTDH) method is an algorithm to solve the time dependent Schrödinger equation which can be considered as a time-dependent version of the MultiConfigurational Self-Consistent Field (MCSCF) method applied to the nu-

80 clei. Within this method the wavefunction $\Psi(Q, t)$ of the system is written as a sum of products of single-particle functions (SPFs), forming a time-dependent orthonormal basis set. SPFs are low dimensional functions: when they contain more than one degree of freedom (DOF), the com${ }_{85}$ bined coordinates $Q_{\kappa} \equiv q_{1, \kappa}, \ldots, q_{d_{\kappa}, \kappa}$ that comprises $d_{\kappa}$ physical DOF are introduced.

The ansatz of the MCTDH wave function reads

$$
\begin{aligned}
\Psi(Q, t) & =\Psi\left(q_{1}, \ldots, q_{f}, t\right) \\
& \equiv \Psi\left(Q_{1}, \ldots, Q_{p}, t\right) \\
& =\sum_{j_{1}}^{n_{1}} \cdots \sum_{j_{p}}^{n_{p}} A_{j_{1}, \ldots, j_{p}}(t) \prod_{\kappa=1}^{p} \varphi_{j_{\kappa}}^{(\kappa)}\left(Q_{\kappa}, t\right) \\
& =\sum_{J} A_{J} \Phi_{J}
\end{aligned}
$$

where $f$ and $p$ denote the number of degrees of freedom and number of combined modes respectively of the system, the $A_{J} \equiv A_{j_{1}, \ldots, j_{p}}$ denotes the MCTDH expansion coefficients and the configuration or Hartree products $\Phi_{J}$ are products of SPFs defined in relation (1). The SPFs are finally represented by linear combinations of time independent primitive basis functions as

$$
\begin{aligned}
\varphi_{j_{\kappa}}^{(\kappa)}\left(Q_{\kappa}, t\right) & =\varphi_{j_{\kappa}}^{(\kappa)}\left(q_{\kappa, 1}, \ldots, q_{\kappa, d_{\kappa}}, t\right) \\
& =\sum_{l_{1} \cdots l_{d}} C_{l_{1} \cdots l_{d}}^{\left(\kappa, j_{\kappa}\right)}(t) \chi_{l_{1}}^{(\kappa)}\left(q_{1, \kappa}\right) \cdots \chi_{l_{d}}^{(\kappa)}\left(q_{d, \kappa}\right),
\end{aligned}
$$

where the primitive functions $\chi_{l_{i}}^{(\kappa)}\left(q_{i, \kappa}\right)$ are usually selected as discrete variable representation (DVR)[16, 17] functions with the time dependent coefficients $C_{l_{1} \cdots l_{d}}^{\left(\kappa, j_{\kappa}\right)}$. The equation of motion for the coefficients and the SPFs are then obtained from the Dirac-Frenkel variational principle. The strength of the method relies in the fact that the number of optimized SPFs used for the propagation is usually much smaller than the number of functions of the primitive basis, resulting in reduced CPU and memory costs.

\section{Atom-Triatom inelastic scattering with MCTDH: Application to $\mathrm{H}_{2} \mathrm{O}$-Ar scattering}

The steps required to perform an inelastic scattering calculation with MCTDH have been described previously 9 10, 11, 12, 18. The basic steps of the procedure will be described in the following (some additional details are found in the above-mentioned publications).

\subsection{Hamiltonian}

Following the derivation of the Kinetic Energy Operator given in Ref. [19], the Hamiltonian of an atom-triatom system in the Body-Fixed (BF) frame with $\mu_{R}$ the reduced mass of the system, $R$ the Atom-Molecule distance and $V_{A t-M o l}$ the Intermolecular Potential Energy Surface can be expressed as

$$
\hat{H}=-\frac{1}{2 \mu_{R}} \frac{\partial^{2}}{\partial R^{2}}+\frac{\hat{\vec{L}}_{R}^{\dagger} \cdot \hat{\vec{L}}_{R}}{2 \mu_{R} R^{2}}+\hat{T}_{M o l}+\hat{V}_{A t-M o l},
$$

where $\vec{L}_{R}$ is the orbital angular momentum of the atomtriatom system and its product with its transpose conjugate expands as

$$
\begin{aligned}
\hat{\vec{L}}_{R}^{\dagger} \cdot \hat{\vec{L}}_{R} & =\hat{\vec{J}}_{B F}^{2}+\hat{\vec{L}}_{M o l, E_{2}}^{2}-2 \hat{J}_{z^{B F}} \hat{L}_{M o l, z^{B F}} \\
& -\hat{J}_{-B F} \hat{L}_{M o l,+^{B F}}-\hat{J}_{+^{B F}} \hat{L}_{M o l,-{ }^{B F}}
\end{aligned}
$$

$\mathrm{BF}$ refers to the Body Fixed frame and $\mathrm{E}_{2}$ to the $\mathrm{E}_{2}$ frame defined in Reference [19]. In the rigid rotor approximation, the kinetic energy operator of the molecule is simply its rigid rotor Hamiltonian which, using the rotational constants $A, B$ and $C$, can be expressed as

$$
\begin{aligned}
\hat{T}_{M o l} & =\left(\frac{A+C}{2}\right) \hat{\vec{L}}_{M o l, B F A}^{2} \\
& +\left[B-\left(\frac{A+C}{2}\right)\right] \hat{\vec{L}}_{M o l, z^{B F A}}^{2} \\
& +\left(\frac{A-C}{4}\right)\left(\hat{\vec{L}}_{M o l,+{ }^{B F A}}^{2}+\hat{\vec{L}}_{M o l,-B F A}^{2}\right),
\end{aligned}
$$

where BFA refers to the Body-Fixed frame of the molecule. In the specific case of the $\mathrm{H}_{2} \mathrm{O}-\mathrm{Ar}$ scattering, the rotational constants $\left(A=27.88063 \mathrm{~cm}^{-1}, B=9.27771 \mathrm{~cm}^{-1}, C=\right.$ $\left.14.52177 \mathrm{~cm}^{-1}\right)[20$ and reduced mass $(\mu=12.41519 A M U)$ were used for the calculations. The PES used in this work was built with the strategies described in recently published papers 21, 22]: it will be presented in a separate publication along with a more detailed description of the conventional time independent calculations. 


\subsection{Initial wavepacket}

Once the Hamiltonian of the system is known, a wave packet defining the initial state of the system has to be constructed: it is in this case a rovibrational state of the molecule (rigid rotor $\mathrm{H}_{2} \mathrm{O}$ ) multiplied by a Gaussian function along the dissociative coordinate $R$ set initially far away from the interaction region. The parameters of the Gaussian are selected such that they cover the energy range of interest for the scattering process. In the Heidelberg MCTDH package 23], the plenerd program is a useful tool that helps to specify the initial wave packet. The Wigner DVR, 24] which has recently been implemented in the Heidelberg MCTDH package by P. S. Thomas [25], was used in our calculations to represent the angular degrees of freedom of the system. To construct the initial state, and also all of the product rotational states after the scattering, we begin using a different Hamiltonian $\hat{H}_{\text {init }}$ written as

$$
\hat{H}_{\text {init }}=-\frac{1}{2 \mu_{R}} \frac{\partial^{2}}{\partial R^{2}}+\hat{T}_{M o l}+\hat{V}_{\text {init }}(R),
$$

115 where the potential term is $\hat{V}_{\text {init }}(R)=\kappa\left(R-R_{0}\right)^{2}$ with $R_{0}$ the starting point of the wavepacket and $\kappa$ a constant correlated to the width of the initial wavepacket. In our application of $\mathrm{H}_{2} \mathrm{O}+\mathrm{Ar}, R_{0}=30$ bohrs, $\kappa=15.3011$ a.u. and the initial impulsion $p=-13$ a.u. with the minus sign indicating that the wavepacket moves towards the interaction region. The wavefunction solution of that Hamiltonian is operated on with an impulsion to impart the initial wavepacket with a particular momentum. The value of the momentum and the width of the initial wavepacket provides the energy distribution of the initial wavepacket, ${ }_{145}$ and also of the final wavefunctions when the Tannor and Weeks method [26] is selected to analyze the propagation. The diagonalization of $H_{\text {int }}$ gives the required wavefunctions whose eigenvalues are the sum of rotational energies of $\mathrm{H}_{2} \mathrm{O}$ combined with harmonic oscillator values in the $\mathrm{e}_{150}$ scattering coordinate. The first few ortho and para rotational states of rigid $\mathrm{H}_{2} \mathrm{O}$ obtained by this procedure (after subtracting the harmonic oscillator eigenvalues) are shown in Table 1 .

\subsection{Complex Absorbing Potential (CAP)}

The next step is to select a suitable CAP to absorb the outgoing wavepacket before the grid boundary: too strong of a CAP will produce reflections of the wavepacket leading to unphysical features in the spectrum and too weak of a160 CAP may not fully absorb the outgoing wavepacket. For a CAP of the form

$$
W(x)=\eta\left|x-x_{c}\right|^{b} \Theta\left(x-x_{c}\right),
$$

the plcap program of the MCTDH package allows one to ${ }^{165}$ optimize the CAP parameters for the scattering energy range of interest. Here $\Theta$ is the Heaviside step function, $x_{c}$ its starting point was selected to be 32 bohrs, $\eta$ its
Table 1: Rotational Energy Levels $\left(\mathrm{cm}^{-1}\right)$ of ortho and para $-\mathrm{H}_{2} \mathrm{O}$. The rotational constants used are $\mathrm{A}=27.88063 \mathrm{~cm}^{-1}, \mathrm{~B}=9.27771$ $\mathrm{cm}^{-1}$ and $\mathrm{C}=14.52177 \mathrm{~cm}^{-1}$.

\begin{tabular}{lccc}
\hline \multicolumn{2}{c}{ ortho- $\mathrm{H}_{2} \mathrm{O}$} & \multicolumn{2}{c}{ para $-\mathrm{H}_{2} \mathrm{O}$} \\
\hline $1_{0,1}$ & 23.7995 & $0_{0,0}$ & 0.0000 \\
$1_{1,0}$ & 42.4024 & $1_{1,1}$ & 37.1583 \\
$2_{1,2}$ & 79.5132 & $2_{0,2}$ & 70.1329 \\
$2_{2,1}$ & 135.3220 & $2_{1,1}$ & 95.2454 \\
$3_{0,3}$ & 136.8897 & $2_{2,0}$ & 136.5876 \\
$3_{1,2}$ & 173.5975 & $3_{1,3}$ & 142.3309 \\
$3_{2,1}$ & 212.6276 & $3_{2,2}$ & 206.7204 \\
$3_{3,0}$ & 287.5373 & $3_{3,1}$ & 287.3396 \\
\hline
\end{tabular}

Table 2: Parameters of the primitive basis used for the improved relaxation and inelastic scattering calculations of $\mathrm{H}_{2} \mathrm{O}-\mathrm{Ar}$. FFT stands for the Fast Fourier Transform. Wigner and Exp stand respectively for Wigner DVR and Exponential DVR. K stands for the momentum representation of the first Euler angle. The distance units is bohrs and the angle is radians.

\begin{tabular}{lcccc}
\hline & $R$ & $\beta_{\mathrm{H}_{2} \mathrm{O}}$ & $\gamma_{\mathrm{H}_{2} \mathrm{O}}$ & $\alpha_{\mathrm{H}_{2} \mathrm{O}}$ \\
\hline Primitive basis & FFT & Wigner & Exp & $\mathrm{K}$ \\
Number of points & 768 & 16 & 31 & 11 \\
Range & $4.0-45.0$ & $0-\pi$ & $0-2 \pi$ & $-5,5$ \\
Size of SPF basis & 45 & & 45 & \\
\hline
\end{tabular}

\subsection{Running the calculations and convergence}

With all of the necessary parameters specified, the calculations can be run while confirming convergence of the primitive and SPF bases, as well as convergence of the calculation with respect to the CAP. Since we are interested in our $\mathrm{H}_{2} \mathrm{O}+\mathrm{Ar}$ scattering application in the low energy range and modest excitations of the rigid $\mathrm{H}_{2} \mathrm{O}$, we selected a relatively compact primitive basis set whose range and the number of SPFs used for the calculation are presented in Table 2. However, even if we were to double the number of primitive basis functions, the number of necessary SPFs (decided in the convergence checking process) will typically only slowly increase, therefore making the calculations affordable. In some cases, it may be possible to compute only a few total angular momentum $\left(J_{B F}\right)$ values, and estimate the probabilities for intermediate $J_{B F}$ by interpolation. Any such interpolation must be done very carefully to ensure that unphysical effects (such as nonzero transition probabilities at closed collisional energies) do not occur.

In the $\mathrm{H}_{2} \mathrm{O}+\mathrm{Ar}$ scattering case, the convergence of the primitive and the SPFs bases as well as the performance of the CAP were all tested. In this study, the employed CAP is broad and its strength is small, hence it handles well the low energy region of the spectrum. The primitive basis selected here is sufficiently complete to describe scattering up to $1500 \mathrm{~cm}^{-1}$ (a generous number of rotational states of rigid $\mathrm{H}_{2} \mathrm{O}$ will be closed at the energy limit to ensure convergence of time independent close coupling cal- 


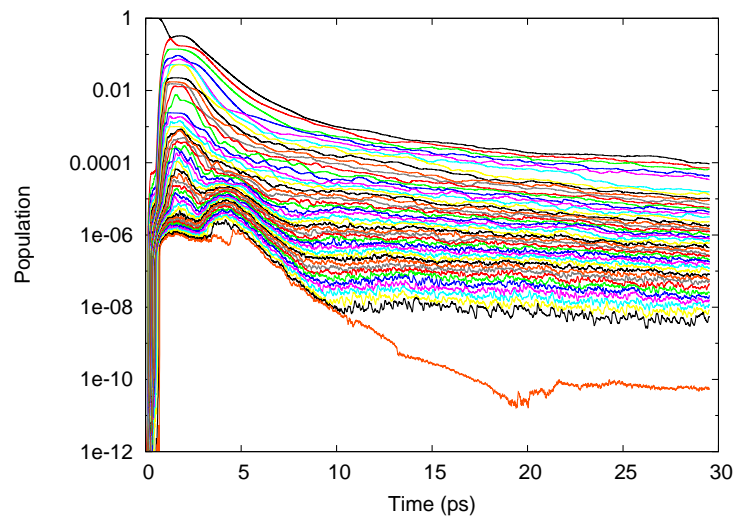

Figure 1: Population of the SPF as a function of time for $J_{B F}=20$.

culations). For the time-dependent MCTDH calculations, grid population analysis tools (rdgpop) implemented in the program indicated that the size of the primitive basis could be reduced without compromising accuracy (as was done for the $K$-mode shown above where we found that ${ }^{22}$ only 11 functions were necessary to describe the scattering). The convergence of the SPF basis can be checked in two ways. One is to perform several calculations with increasing numbers of SPFs and check the convergence of the resulting probabilities for the transitions of interest. Another is to check the (natural) population of the lowest populated SPF through the propagation and ensure that it is smaller than a specified tolerance: here we chose a tight value of $10^{-6}$. Figure 1 shows the population of the $45 \mathrm{SPFs}$ as a function of time for $J_{B F}=20$. From the plot we deduce that we likely could have specified 5 to $_{225}$ 10 fewer SPFs without a significant loss in accuracy. We provide Supporting Information 27] confirming the convergence with respect to the number of SPFs.

The propagation times for these calculations are quite long, from $30 \mathrm{ps}$ for $J_{B F}=0$ down to 5 ps for $J_{B F}=150$. This is due to our interest in the low energy range $[0,0.3]^{23}$ $\mathrm{eV}$, and that fact that we specified that more than $99 \%$ of the wavepacket should be absorbed by the CAP to complete the propagation. Additional test calculations done on a larger energy range (up to $1 \mathrm{eV}$ for example) required a much shorter propagation time for the same fraction of the wavepacket to be absorbed (during a calculation, the low energy part of the wave packet takes the longest time to reach the CAP where it gets absorbed).

\subsection{Analysis of the results}

Once the propagation is complete, either the flux 28, 29, 14] or the Tannor and Weeks [26] method is used to obtain the probabilities for the various transitions. We tested both methods but the results presented are those from the ${ }_{245}$ Tannor and Weeks method. While the two methods lead to similar probabilities, the flux method usually produces a small, but non-negligible flux in an energetically forbidden region (below the total energy threshold). This numerical issue can easily be corrected by zeroing the probabilities in the energetically forbidden region when summing the cross-sections (eq. 8). Conversely, the Tannor and Weeks method requires a broad energy distribution of the final wavefunctions (wavefunctions on which the propagated wavefunction is projected) to make the correlation 25 function disappear more quickly.

A weighted sum of the probabilities obtained from the flux or Tannor and Weeks approach then produces the inelastic cross-section from the relation

$$
\begin{aligned}
\sigma_{j_{0} l_{0}}^{j l}(E)= & \frac{\pi \hbar^{2}}{2 \mu_{R}\left(2 j_{0}+1\right) E_{c o l l}} \sum_{J_{B F}=0}^{\infty}\left(2 J_{B F}+1\right) \times \\
& \sum_{K} P_{j l \leftarrow j_{0} l_{0}}^{J_{B F}}(E),
\end{aligned}
$$

where $j$ is the orbital angular momentum quantum number of the molecule, $K$ and $l$ are respectively its projection along the Space-Fixed axis and an index running from $-j$ to $j$. In the literature, $l$ is often replaced by $K_{a} K_{c}$ (as it is done in Table 1 where $K_{a}$ and $K_{c}$ are respectively the projection of the molecular orbital angular momentum along the Body-Fixed (Molecular) axis in the prolate and oblate limits.

The cross-sections can then be integrated to obtain the rates from the formula

$$
k_{f i}(T)=\sqrt{\frac{8 \beta^{4}}{\pi \mu_{R} \beta}} \int_{0}^{\infty} d E_{\text {coll }} e^{-\beta E_{\text {coll }}} E_{\text {coll }} \sigma_{f i}(E),
$$

where $\beta=1 / k T$ with $k$ being the Boltzmann constant. The subscripts $i, f$ replace a rovibrational state $j K_{a} K_{c}$ as specified above and the collinear energy is $E_{\text {coll }}=E-\epsilon_{j_{0} l_{0}}$ with $E$ the total energy and $\epsilon_{j_{0} l_{0}}$ the energy of the initial rovibrational state.

We performed for comparison (rigid-rotor) time independent close-coupling calculations using the quantum scattering code MOLSCAT 30. We used the same rotational constants and reduced mass as for the MCTDH calculations and the rotational basis was generously selected so as to converge the calculations up to $1500 \mathrm{~cm}^{-1}$. Figure 2 shows the rotational excitation cross-sections from the $0_{00}$ state to the excited para-states up to $j=3$ computed using the MCTDH method compared with the corresponding time independent close coupling calculations using the MOLSCAT 30] code. Single core calculations for 10 energy to points took several days (up to few weeks for higher energy values) to run with the MOLSCAT code. The excellent shared-memory parallelization of MCTDH made it possible to run 16-core calculations for a specific value of $J_{B F}$ in a maximum of 3 days for the longest propagation: the time was significantly shorter for propagations at higher $J_{B F}$. The calculations were done for 80 selected intermittent values of $J_{B F}$ between 0 and 120 , and the remaining values 
were obtained by interpolation using the $J$-interpolation algorithm presented in Appendix A of Reference 9. We present in Supporting Information 27 the close correspondence between some interpolated and computed transition probabilities. Figure 2 illustrates the close agreement that was obtained between the time independent and the time dependent MCTDH calculations. There are some slight differences, but the shape and general magnitudes of the cross sections are very similar, even for cross-sections with low intensities (see Figure 2 (b)). Small differences between time-independent and time-dependent treatments (especially at the lowest collision energies) have been reported previously for reactive scattering. 31, 32 Here, considering the convergence of the calculations, we speculate that some of the differences between the time-independent and MCTDH cross-sections may arise from interpolation between $J_{B F}$-values. Perhaps even closer agreement could be achieved by performing additional calculations at the missing $J_{B F}$ values, extending the range of the dissociative coordinates and expanding the PES with spherical harmonics as it was done for the time independent calculations instead of using POTFIT as it is done here. Overall, we are satisfied with the level of agreement, since the rates obtained by integrating the MCTDH and MOLSCAT cross-sections differ by less than $5 \%$.

\section{Conclusion}

We present a straightforward procedure to perform efficient inelastic scattering calculations between an atom and a triatomic molecule using the MCTDH method as implemented in the Heidelberg MCTDH package. The $\mathrm{H}_{2} \mathrm{O}-\mathrm{Ar}$ collision system is treated with $\mathrm{MCTDH}$ and compared with time independent close coupling calculations done on the same PES. Excellent agreement between time independent and MCTDH calculations over a wide energy range provides further evidence that the MCTDH approach is reliable to study those processes even at low collision energies. These results, combined with the well known favorable scalability with increased system dimensionality indicates $\mathrm{MCTDH}$ as a worthwhile approach to study inelastic scattering processes in more complex cases (with w $_{300}$ high densities of states and dimensionality) where computational costs are often prohibitive. Although there has been impressive progress with semiclassical or mixed quantum/classical methods, an accurate fully quantum mechanical description is required to serve as a benchmark. ${ }^{305}$ MCTDH is well-positioned to be the method of choice in many cases for comparisons with experiments where transitions from a specific initial state are of interest.

\section{Acknowledgements}

This work was funded by the US National Science Foundation (CHE-1566246 to RD).
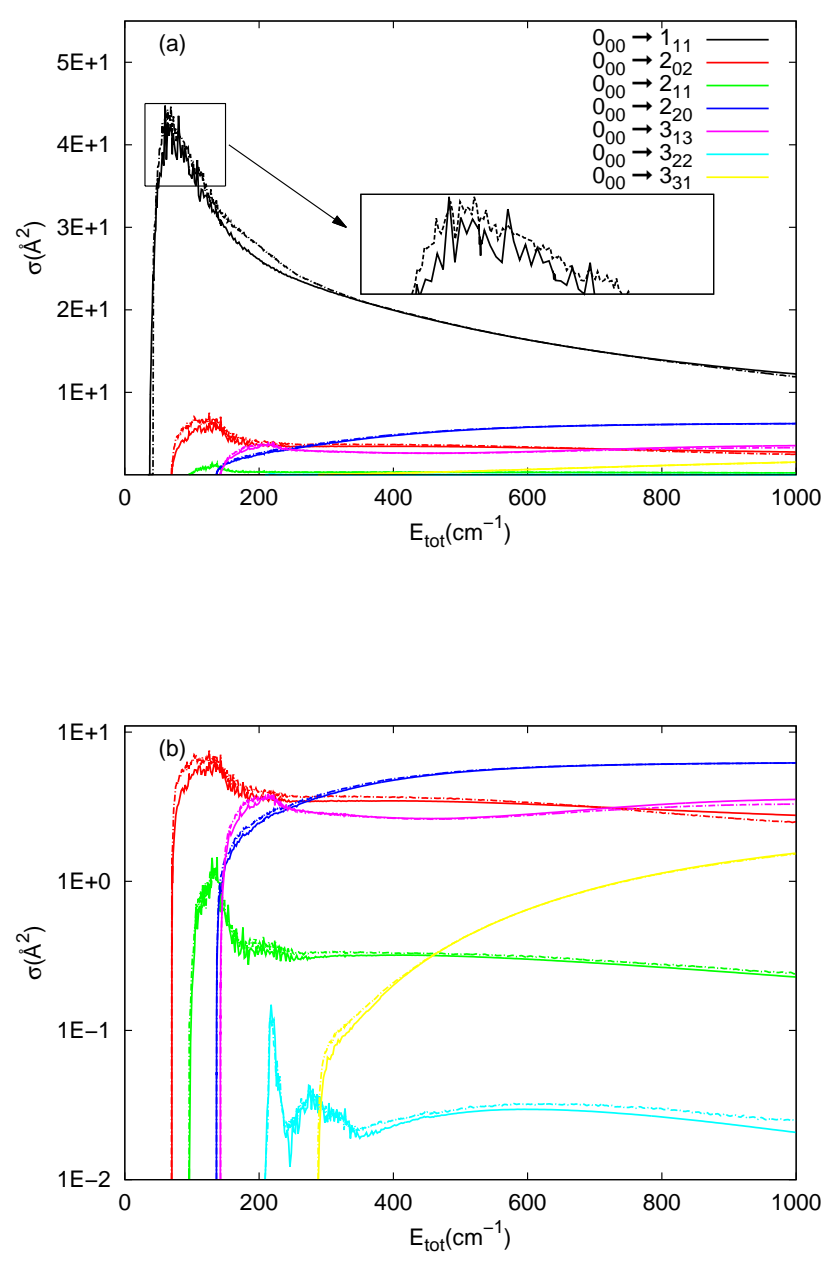

Figure 2: Inelastic scattering cross-sections computed using the MCTDH method (dot-dashed lines) and the time independent close coupling approach (full lines). (a) Linear scale, (b) Logarithmic scale. The inset in figure (a) shows a zoom of the squared section.

\section{References}

\section{References}

[1] G. W. Flynn, C. S. Parmenter, A. M. Wodtke, Vibrational energy transfer, J. Phys. Chem. 100 (31) (1996) 12817-12838.

[2] J. D. Steill, A. W. Jasper, D. W. Chandler, Determination of the collisional energy transfer distribution responsible for the collision-induced dissociation of $\mathrm{NO}_{2}$ with Ar, Chem. Phys. Lett. 636 (2015) 1-14.

[3] D. A. Babikov, A. Semenov, Recent Advances in Development and Applications of the Mixed Quantum/Classical Theory for Inelastic Scattering, J. Phys. Chem. A 120 (2015) 319-331.

[4] L. Wiesenfeld, W. F. Thi, P. Caselli, A. Faure, L. Bizzocchi, J. Brando, D. Duflot, E. Herbst, S. J. Klippenstein, T. Komatsuzaki, C. Puzzarini, O. Roncero, H. Teramoto, M. Toda,

A. van der Avoird, H. Waalkens, Theory of Gas Phase Scattering and Reactivity for Astrochemistry|arXiv: 1610.00438. URL http://arxiv.org/abs/1610.00438

[5] S. A. Ndengué, R. Dawes, F. Gatti, H. D. Meyer, Resonances of HCO Computed Using an Approach Based on the Multicon- 
figuration Time-Dependent Hartree Method, J. Phys. Chem. A 119 (2015) 12043-12051. bilities by a minimal number of wavepackets, Chem. Phys. Lett. 241 (5) (1995) 497-501.

[7] R. Welsch, F. Huarte-Larrañaga, U. Manthe, State-to-state reaction probabilities within the quantum transition state frame-395 work, J. Chem. Phys. 136 (6) (2012) 064117.

[8] R. Welsch, U. Manthe, Loss of memory in $\mathrm{h}+\mathrm{ch} 4 \rightarrow \mathrm{h} 2+\mathrm{ch} 3$ state-to-state reactive scattering, J. Phys. Chem. Lett. 6 (3) (2015) 338-342.

[9] F. Gatti, F. Otto, S. Sukiasyan, H. D. Meyer, Rotational Ex-400 citation Cross Sections of Para- $\mathrm{H}_{2}+$ Para- $\mathrm{H}_{2}$ Collisions. A FullDimensional Wave-Packet Propagation Study Using an Exact Form of the Kinetic Energy., J. Chem. Phys. 123 (2005) 174311.

[10] F. Otto, F. Gatti, H. D. Meyer, Rotational Excitations in Para$\mathrm{H}_{2}+$ Para $-\mathrm{H}_{2}$ Collisions: Full- and Reduced-Dimensional Quan-405 tum Wave Packet Studies Comparing Different Potential Energy Surfaces., J. Chem. Phys. 128 (2008) 064305.

[11] F. Otto, F. Gatti, H.-D. Meyer, Erratum: "Rotational excitations in para $-\mathrm{H}_{2}+$ para $-\mathrm{H}_{2}$ collisions: Full- and reduceddimensional quantum wave packet studies comparing different potential energy surfaces", J. Chem. Phys. 131 (2009) 049901.

[12] R. F. Malenda, F. Gatti, H. D. Meyer, D. Talbi, A. P. Hickman, Comparison of the Multi-Configuration, Time-Dependent Hartree (MCTDH) Method with the Arthurs and Dalgarno Coupled-Channel Method for Rotationally Inelastic Scattering., Chem. Phys. Lett. 585 (2013) 184.

[13] H. D. Meyer, U. Manthe, L. S. Cederbaum, The MultiConfigurational Time-Dependent Hartree Approach, Chem. Phys. Lett. 165 (1990) 73-78.

[14] M. H. Beck, A. Jäckle, G. A. Worth, H. D. Meyer, The multiconfiguration time-dependent Hartree $(\mathrm{MCTDH})$ method: a highly efficient algorithm for propagating wavepackets, Phys. Rep. 324 (2000) 1.

[15] H. D. Meyer, F. Gatti, G. A. Worth (Eds.), Multidimensional Quantum Dynamics: MCTDH Theory and Applications, WileyVCH: Weinheim, 2009.

[16] J. Echave, D. C. Clary, Potential optimized discrete variable representation, Chem. Phys. Lett. 190 (1992) 225.

[17] H. Wei, T. Carrington, The discrete variable representation of a triatomic Hamiltonian in bond length-bond angle coordinates, J. Chem. Phys. 97 (1992) 3029.

[18] S. A. Ndengue, R. Dawes, F. Gatti, Rotational Excitations in CO-CO Collisions at Low Temperature: Time-Independent and Multiconfigurational Time-Dependent Hartree Calculations, J. Phys. Chem. A 119 (2015) 7712.

[19] F. Gatti, F. Iung, Exact and constrained kinetic energy operators for polyatomic molecules: The polyspherical approach, Phys. Rep. 1 (2009) 484.

[20] F. C. DeLucia, P. Helminger, W. H. Kirchhoff, Microwave spectra of molecules of astrophysical interest V. Water vapor, J. Phys. Chem. Ref. Data 3 (1) (1974) 211-219.

[21] M. Majumder, S. Ndengue, R. Dawes, Automated construction of potential energy surfaces, Mol. Phys. 114 (1) (2016) 1-18.

[22] R. Dawes, S. A. Ndengué, Single-and multireference electronic structure calculations for constructing potential energy surfaces, Int. Rev. Phys. Chem. 35 (3) (2016) 441-478.

[23] G. A. Worth, M. H. Beck, A. Jäckle, H.-D. Meyer, The MCTDH Package, Version 8.2, (2000). H.-D. Meyer, Version 8.3 (2002), Version 8.4 (2007). Current version: 8.4 .12 (2016). See http://mctdh.uni-hd.de/.

[24] C. Leforestier, Grid method for the Wigner functions. Application to the van der Waals system $\mathrm{Ar}-\mathrm{H}_{2} \mathrm{O}$, J. Chem. Phys. 101 (9) (1994) 7357-7363.

[25] G. Füchsel, P. Thomas, J. den Uyl, F. N. Y. Öztürk, H.-D. Meyer, G.-J. Kroes, Rotational effects on the dissociation dynamics of $\mathrm{CHD}_{3}$ on $\mathrm{Pt}(111)$, Phys. Chem. Chem. Phys. 18 (2016) 8174-8185. doi:10.1039/c5cp07898a.

[26] D. J. Tannor, D. E. Weeks, Wave Packet Correlation Function Formulation of Scattering Theory: The Quantum Analog of

Classical S-Matrix Theory, J. Chem. Phys. 98 (1993) 3884-3893.

[27] See supporting informations document No.

[28] T. Seideman, W. H. Miller, Quantum Mechanical Reaction Probabilities via a Discrete Variable Representation-Absorbing Boundary Condition Green's Function, J. Chem. Phys. 97 (1992) 2499-2514.

[29] A. Jäckle, H.-D. Meyer, Time-dependent calculation of reactive flux employing complex absorbing potentials: General aspects and application within MCTDH, J. Chem. Phys. 105 (1996) 6778 .

[30] J. M. Hutson, S. Green, MOLSCAT, ver. 14, distributed by Collabor. Comp. Proj. 6, Daresbury lab, UK, Eng. Phys. Sci. Res. Council (1994).

[31] Z. Sun, D. Yu, W. Xie, J. Hou, R. Dawes, H. Guo, Kinetic isotope effect of the ${ }^{16} \mathrm{O}+{ }^{36} \mathrm{O}_{2}$ and ${ }^{18} \mathrm{O}+{ }^{32} \mathrm{O}_{2}$ isotope exchange reactions: Dominant role of reactive resonances revealed by an accurate time-dependent quantum wavepacket study, J. Chem. Phys. 142 (17) (2015) 174312.

[32] T. R. Rao, G. Guillon, S. Mahapatra, P. Honvault, Quantum dynamics of ${ }^{16} \mathrm{O}+{ }^{36} \mathrm{O}_{2}$ and ${ }^{18} \mathrm{O}+{ }^{32} \mathrm{O}_{2}$ exchange reactions, J. Chem. Phys. 142 (17) (2015) 174311. 


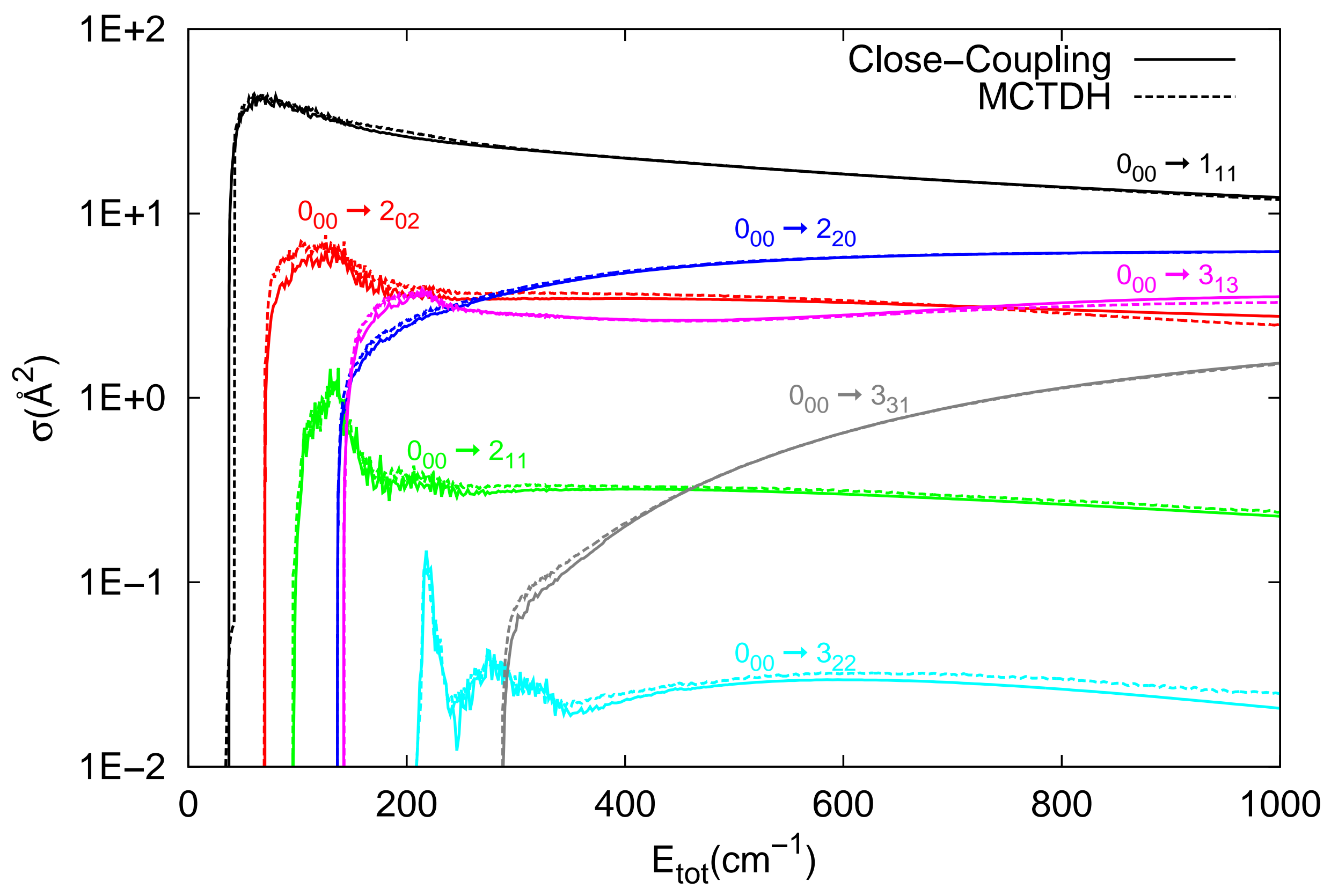

